\title{
Entanglement for multipartite systems of indistinguishable particles *
}

\author{
Janusz Grabowski ${ }^{\dagger}$ \\ Polish Academy of Sciences, Institute of Mathematics, \\ Śniadeckich 8, P.O. Box 21, 00-956 Warsaw, Poland \\ Marek Kuś ${ }^{\ddagger}$ \\ Center for Theoretical Physics, Polish Academy of Sciences, \\ Aleja Lotników 32/46, 02-668 Warszawa, Poland \\ Giuseppe Marmo ${ }^{\S}$ \\ Dipartimento di Scienze Fisiche, Università "Federico II" di Napoli \\ and Istituto Nazionale di Fisica Nucleare, Sezione di Napoli, \\ Complesso Universitario di Monte Sant Angelo, \\ Via Cintia, I-80126 Napoli, Italy
}

\begin{abstract}
We analyze the concept of entanglement for multipartite system with bosonic and fermionic constituents and its generalization to systems with arbitrary parastatistics. We use the representation theory of symmetry groups to formulate a unified approach to this problem in terms of simple tensors with appropriate symmetry. For an arbitrary parastatistics, we define the S-rank generalizing the notion of the Schmidt rank. The S-rank, defined for all types of tensors, serves for distinguishing entanglement of pure states. In addition, for Bose and Fermi statistics, we construct an analog of the Jamiołkowski isomorphism.
\end{abstract}

MSC 2000: 81P16 (Primary); 15A69, 81R05 (Secondary).

Key words: entanglement, tensor product, symmetry group, Bose statistics, Fermi statistics, parastatistics, Young diagram, Segre map, Jamiołkowski isomorphism.

PACS: 03.65.Aa, 03.67.Mn, 02.10.Xm.

\section{Introduction}

Confronted with entangled states of a bipartite system, Schrödinger said that entanglement was not ...one but rather the characteristic trait of quantum mechanics, the one that enforces its entire departure from classical line of thought... [1].

\footnotetext{
* Research of the first two authors financed by the Polish Ministry of Science and Higher Education under the grant No. N N202 090239.

†email: jagrab@impan.pl

‡email: marek.kus@cft.edu.pl

§email: marmo@na.infn.it
} 
Note that Schrödinger's interests for this aspect of Quantum Mechanics originated from the EPR paper. Indeed, the entanglement for states of composite systems created quite an embarrassment for theoretical physicists not sharing the point of view of the "Copenhagen interpretation" of quantum mechanics. As a matter of fact, the entanglement played a rather important role in the development of quantum mechanics, as it obliged the physics community to face the nonlocal nature of the description of natural processes. Nowadays, however, entanglement is considered as an important resource for quantum computation, quantum information and quantum teleportation.

The usual definition of entangled states is given by defining first separable pure states as decomposable tensor products and then declaring a state to be entangled if it is not separable.

In the case of a system containing identical constituents, the problem of the definition of entanglement has to be reconsidered, since the indistinguishability produces some amount of entanglement by its own. For instance, a non-zero skew-symmetric 2-tensor is never decomposable. We cannot therefore simply transpose to the Hilbert space of the composite system the requirement that non-entanglement is guaranteed by the factorization of the total state into a simple tensor product of vectors corresponding to the two subsystems. We have to analyze better the meaning of entanglement per se and refine the concept of separability.

Several nonequivalent ways of identification and quantification of the phenomenon were proposed. In 22 the authors explicitly stated the problem of distinguishing non-local correlations from those caused by the Pauli principle measured in fundamental experiments, aiming at checking the validity of quantum mechanics involving two identical fermions. To quantify the amount of extra correlations, the authors used the number of terms in the decomposition of the wave function in terms of elementary $2 \times 2$ 'Slater determinants' (see Section 6 below). Similar ideas, based on the lengths of the canonical decomposition of the wave function, were used in [3] for description of correlations in double ionization of atoms in strong electromagnetic fields.

A scheme of extracting the correlations caused by the Pauli principle in fermionic systems in order to characterize a genuine entanglement, became important in analysis of elementary quantum gates based on quantum dots [4. Subsystems here are no longer separated by macroscopic distances; on the contrary, they occupy the same spatial regions and their indistinguishability becomes relevant. The problem was analyzed in [5] and further in [6] in terms of modern theory of entanglement. Measures of correlations were constructed in analogy with the distinguishable particles case, again by employing algebraic properties of the coefficient matrix in the expansion of a state in terms of basis states.

Similar ideas can be applied, and in fact were applied, to bosons [6, 7]. In the bosonic case non-entangled states are again identified with simple symmetric tensors (see Section 4 below). Thus, in the case of two bosons a state is non-entangled if and only if it is a tensor product of two identical one-particle states. This definition should be contrasted with another one advocated already in [8], where bosonic and fermionic systems were treated in parallel. To identify two partite systems with minimal identical-particle correlations, i.e., without entanglement, one considers a situation when two particles are confined to two non-overlapping spatial regions in which they are subjected to spatially confined independent measurements. The correlations are minimal if, for every independent choice of measurements, the probability of outcomes factorizes into the product of single particles probabilities of outcomes. For two fermions, the result reduces to the demand that the wave function of the whole system is a simple antisymmetric tensor (a single Slater determinant), $|\Psi\rangle \sim(|\psi\rangle \otimes|\phi\rangle-|\phi\rangle \otimes|\psi\rangle)$, i.e., to the very definition adopted by other authors cited above. For bosons, however, the definition leads to two different classes of minimally correlated state. In addition to tensor products of identical one-particle 
states, also wave functions in the form of Slater permanents i.e., symmetrized tensor products of orthogonal one-particle states, $|\Psi\rangle \sim(|\psi\rangle \otimes|\phi\rangle+|\phi\rangle \otimes|\psi\rangle),\langle\psi \mid \phi\rangle=0$, are classified as minimally entangled 1$]$ The definition can be easily extended to many particle systems [9].

In a series of papers by Ghirardi et. al. [10, 11, 12, a definition of non-entangled state was based on the concept of a complete set of properties possessed by a constituent of a composite system. For a bi-partite system, in a pure state $\rho$, we say that a one of the constituents has a complete set of properties if and only if there exists a rank-one projection operator $P$ acting in a single particle space $\mathcal{H}$ such that $\operatorname{Tr}(E \rho)=1$, where $E=P \otimes I+I \otimes P-P \otimes P$ is a projection operator acting in $\mathcal{H} \wedge \mathcal{H}$ or $\mathcal{H} \vee \mathcal{H}$ for, respectively, fermions and bosons. This idea resembles the one advocated in the above cited papers of Herbut, but rather than invoking directly 'local' measurements it stresses properties of subsystem states. The result is the same. For fermions, non-entangled states are antisymmetrized tensor products, whereas for bosons they split into two classes: products of two identical states, or symmetrized products of two orthogonal states. Whereas for fermions the criterion of non-entanglement, based on the number of coefficients in expansion in terms of elementary $2 \times 2$ Slater determinants, remains functional, it is not the case for bosons. Here, states expressible as linear combinations of two Slater permanents are non-entangled if the both coefficients of expansion are the same - such states can be expressed as symmetrized products of two orthogonal vectors [9, 10]. A concept of entanglement for indistinguishable particles based on a measurability of correlations was recently considered also in 13 .

In this paper we attempt to approach this problem from a mathematical view point, where structures and available mathematical constructions are used as a guide. A fundamental character of our work depends also on reviewing and generalizing basic concepts of mathematical foundations in understanding entanglement. We shall provide few physical considerations in the conclusions, where we also briefly compare our results with other outlined above. Here, let us mention only that our natural and unifying mathematical model strongly suggests nonentanglement for bosons to be associated with tensor products of identical states.

The paper is organized in the following way. In Section 2 we recall definitions and basic facts from tensor algebra underlying our analysis of entanglement for multipartite bosonic and fermionic systems. In Section 3 we introduce the concept of duality and analyze contractions between dual spaces of tensors, which allows us to define the S-rank of a tensor, generalizing the Schmidt rank, and then the simplicity of a tensor in Section 4 . Section 5 contains various characterizations of simplicity of a tensor in general, and in the bosonic or fermionic class. In Section 6 we define entanglement for bosonic and fermionic multipartite states and provide a simple characterization of entanglement for pure states in terms of bilinear functions in coefficients of representing tensors. In analysis of entanglement of distinguishable particles, the so called Jamiołkowski isomorphism played the role of a very useful tool. We give an extension of it for boson and fermions in Section 7. The mathematically rigorous generalization of entanglement to multipartite system with arbitrary parastatistics, another novel invention in this paper, is given in Section 8 .

Note that such an unifying approach allowed also for description of pure non-entangled states as the images of generalized Segre maps, in full analogy with the case of distinguishable particles [14, 15]. These questions, however, we decided to postpone to a separate paper.

\footnotetext{
${ }^{1}$ To make the non-entanglement condition in bosonic and fermionic systems completely parallel, one can demand orthogonality also in the latter case, since it does not play a role for fermions.
} 


\section{Tensor algebras}

To describe some properties of systems composed of indistinguishable particles and to fix the notation, let us start with introducing corresponding tensor algebras associated with a Hilbert space $\mathcal{H}$. For simplicity, we assume that $\mathcal{H}$ is finite-, say, $n$-dimensional, but a major part of our work remains valid also for Hilbert spaces of infinite dimensions. Note only that in the infinite dimensions the corresponding tensor product $\mathcal{H}_{1} \otimes \mathcal{H}_{2}$ is the tensor product in the category of Hilbert spaces, i.e., corresponding to Hilbert-Schmidt norm.

In the tensor power $\mathcal{H}^{\otimes k}=\underbrace{\mathcal{H} \otimes \cdots \otimes \mathcal{H}}_{k \text {-times }}$, we distinguish the subspaces: $\mathcal{H}^{\vee k}=\underbrace{\mathcal{H} \vee \cdots \vee \mathcal{H}}_{k \text {-times }}$ of totally symmetric tensors, and $\mathcal{H}^{\wedge k}=\underbrace{\mathcal{H} \wedge \cdots \wedge \mathcal{H}}_{k-\text { times }}$ of totally antisymmetric ones, together with the symmetrization, $\pi_{k}^{\vee}: \mathcal{H}^{\otimes k} \rightarrow \mathcal{H}^{\vee k}$, and antisymmetrization, $\pi_{k}^{\wedge}: \mathcal{H}^{\otimes k} \rightarrow \mathcal{H}^{\wedge k}$, projectors:

$$
\begin{aligned}
& \pi_{k}^{\vee}\left(f_{1} \otimes \cdots \otimes f_{k}\right)=\frac{1}{k !} \sum_{\sigma \in S_{k}} f_{\sigma(1)} \otimes \cdots \otimes f_{\sigma(k)}, \\
& \pi_{k}^{\wedge}\left(f_{1} \otimes \cdots \otimes f_{k}\right)=\frac{1}{k !} \sum_{\sigma \in S_{k}}(-1)^{\sigma} f_{\sigma(1)} \otimes \cdots \otimes f_{\sigma(k)} .
\end{aligned}
$$

Here, $S_{k}$ is the group of all permutations $\sigma:\{1, \ldots, k\} \rightarrow\{1, \ldots, k\}$, and $(-1)^{\sigma}$ denotes the sign of the permutation $\sigma$. Note that with every permutation $\sigma \in S_{k}$ there is a canonically associated unitary operator $U_{\sigma}$ on $\mathcal{H}^{\otimes k}$ defined by

$$
U_{\sigma}\left(f_{1} \otimes \cdots \otimes f_{k}\right)=f_{\sigma(1)} \otimes \cdots \otimes f_{\sigma(k)},
$$

so that the map $\sigma \mapsto U_{\sigma}$ is an injective unitary representation of $S_{k} \rightarrow U\left(\mathcal{H}^{\otimes k}\right)$. We will write simply $\sigma$ instead of $U_{\sigma}$, if no misunderstanding is possible. Symmetric and skew-symmetric tensors are characterized in terms of this unitary action by $\sigma(v)=v$ and $\sigma(v)=(-1)^{\sigma} v$, respectively, for all $\sigma \in S_{k}$

We put, by convention, $\mathcal{H}^{\otimes 0}=\mathcal{H}^{\vee 0}=\mathcal{H}^{\wedge 0}=\mathbb{C}$. It is well known that the obvious structure of a unital graded associative algebra on the graded space $\mathcal{H}^{\otimes}=\underset{k=0}{\otimes} \mathcal{H}^{\otimes k}$ (the tensor algebra) induces canonical unital graded associative algebra structures on the spaces $\mathcal{H}^{\vee}=\underset{k=0}{\infty} \mathcal{H}^{\vee k}$ (called the bosonic Fock space) and $\mathcal{H}^{\wedge}=\bigoplus_{k=0}^{\infty} \mathcal{H}^{\wedge k}$ (called the fermionic Fock space) of symmetric and antisymmetric tensors, with the multiplications

$$
\begin{aligned}
v_{1} \vee v_{2} & =\pi^{\vee}\left(v_{1} \otimes v_{2}\right), \\
w_{1} \wedge w_{2} & =\pi^{\wedge}\left(w_{1} \otimes w_{2}\right) .
\end{aligned}
$$

Here, of course,

$$
\pi^{\vee}=\bigoplus_{k=0}^{\infty} \pi_{k}^{\vee}: \mathcal{H}^{\otimes} \rightarrow \mathcal{H}^{\vee}
$$

and

$$
\pi^{\wedge}=\underset{k=0}{\oplus} \pi_{k}^{\wedge}: \mathcal{H}^{\otimes} \rightarrow \mathcal{H}^{\wedge}
$$

are the symmetrization and antisymmetrization projections. Note that the multiplication in $\mathcal{H}^{\vee}$ is commutative, $v_{1} \vee v_{2}=v_{2} \vee v_{1}$, and the multiplication in $\mathcal{H}^{\wedge}$ is graded commutative, $w_{1} \wedge w_{2}=(-1)^{k_{1} \cdot k_{2}} w_{2} \wedge w_{1}$, for $w_{i} \in \mathcal{H}^{\wedge k_{i}}$. 
Denote with $\mathcal{H}^{*}$ the complex dual space of $\mathcal{H}$. The Hermitian product $\langle\cdot \mid \cdot\rangle$ on $\mathcal{H}$ induces a canonical bijection between $\mathcal{H}$ and $\mathcal{H}^{*}$ which, in the Dirac's notation, reads

$$
\mathcal{H} \ni|x\rangle \mapsto\langle x| \in \mathcal{H}^{*} .
$$

We must stress that this is not an isomorphism of complex linear spaces, since the above map is anti-linear. Note, however, that the symmetric tensor algebra $\mathcal{H}^{\vee}$ can be canonically identified with the algebra $\operatorname{Pol}\left(\mathcal{H}^{*}\right)$ of polynomial functions on the complex dual $\mathcal{H}^{*}$ of $\mathcal{H}$. Indeed, any $f \in \mathcal{H}$ can be identified with a linear function $x_{f}$ on $\mathcal{H}^{*}$ by means of the canonical pairing $\langle\rangle:, \mathcal{H} \times \mathcal{H}^{*} \rightarrow \mathbb{C}$ between the dual spaces, as $x_{f}(y)=\langle f, y\rangle$ (we must distinguish this pairing from the Hermitian product on $\mathcal{H}$ ). This can be extended to an isomorphism of commutative algebras in which $f_{1} \vee \cdots \vee f_{k}$ corresponds to the homogenous polynomial $x_{f_{1}} \cdots x_{f_{k}}$. Similarly, one identifies $\mathcal{H}^{\wedge}$ with the Grassmann algebra $\operatorname{Grass}\left(\mathcal{H}^{*}\right)$ of polynomial (super)functions on $\mathcal{H}^{*}$. Here, however, with $f \in \mathcal{H}$ we associate a linear function $\xi_{f}$ on $\mathcal{H}^{*}$ regarded as and odd function: $\xi_{f} \xi_{f^{\prime}}=-\xi_{f^{\prime}} \xi_{f}$. In the language of supergeometry one speaks about the purely odd manifold $\Pi \mathcal{H}^{*}$ obtained from the standard (purely even) linear manifold $\mathcal{H}^{*}$ by changing the parity of linear functions. In this sense, $\mathcal{H}^{\wedge}$ is the algebra of holomorphic (super)functions on the complex supermanifold $\mathcal{H}^{*}$.

If we fix a basis $e_{1}, \ldots, e_{n}$ in $\mathcal{H}$ and associate with its elements even linear functions $x_{1}, \ldots, x_{n}$ on $\mathcal{H}^{*}$, and odd linear functions $\xi_{1}, \ldots, \xi_{n}$ on $\Pi \mathcal{H}^{*}$, then $\mathcal{H}^{\vee} \simeq \mathbb{C}\left[x_{1}, \ldots, x_{n}\right]$, i.e. $\mathcal{H}^{\vee}$ becomes isomorphic with the algebra of complex polynomials in $n$ commuting variables. Similarly, $\mathcal{H}^{\wedge} \simeq$ $\mathbb{C}\left[\xi_{1}, \ldots, \xi_{n}\right]$, i.e., $\mathcal{H}^{\wedge}$ is isomorphic with the algebra of complex Grassmann polynomials in $n$ anticommuting variables. The subspaces $\mathcal{H}^{\vee k}$ and $\mathcal{H}^{\wedge k}$ correspond to homogenous polynomials of degree $k$. It is straightforward that homogeneous polynomials $x_{1}^{k_{1}} \cdots x_{n}^{k_{n}}$, with $k_{1}+\cdots+$ $k_{n}=k$, form a basis of $\mathcal{H}^{\vee k}$, while homogeneous Grassmann polynomials $\xi_{i_{1}} \wedge \cdots \wedge \xi_{i_{k}}$, with $1 \leq i_{1}<i_{2}<\cdots<i_{k} \leq n$, form a basis of $\mathcal{H}^{\wedge k}$. In consequence, $\operatorname{dim} \mathcal{H}^{\vee k}=\left(\begin{array}{c}n+k-1 \\ k\end{array}\right)$ and $\operatorname{dim} \mathcal{H}^{\wedge k}=\left(\begin{array}{l}n \\ k\end{array}\right)$, so the gradation in the fermionic Fock space is finite (for finite-dimensional $\mathcal{H}$ ). Of course, we can put together both algebras and consider the tensor product $\mathcal{H}_{1}^{\vee} \otimes \mathcal{H}_{2}^{\wedge}$, with $\operatorname{dim} H_{1}=n$ and $\operatorname{dim} H_{2}=m$, which is a graded associative algebra with a bi-gradation $\mathbb{N} \times \mathbb{N}$ concentrated in $\mathbb{N} \times\{0,1, \ldots, m\}$,

$$
\mathcal{H}_{1}^{\vee} \otimes \mathcal{H}_{2}^{\wedge}=\bigoplus_{(k, l) \in \mathbb{N} \times\{0,1, \ldots, m\}} \mathcal{H}_{1}^{\vee k} \otimes \mathcal{H}_{2}^{\wedge l} .
$$

Here, $\mathcal{H}_{1}^{\vee k} \otimes \mathcal{H}_{2}^{\wedge l}$ represent systems composed of $k$ bosons, described by a Hilbert space of dimension $n$, and $l$ fermions in the Hilbert space of dimension $m$. The whole algebra $\mathcal{H}_{1}^{\vee} \otimes \mathcal{H}_{2}^{\wedge}$ is the algebra of polynomial functions on the linear supermanifold $\mathcal{H}_{1}^{*} \times \Pi \mathcal{H}_{2}^{*}$ of dimension $(n, m)$. Such functions are written as finite complex linear combinations

$$
\sum_{\substack{k_{1}, \ldots, k_{n} \\ 1 \leq i_{1}<\cdots<i_{l} \leq m}} a_{k_{1}, \ldots, k_{n}}^{i_{1}, \ldots, i_{l}} x_{1}^{k_{1}} \cdots x_{n}^{k_{n}} \xi_{i_{1}} \cdots \xi_{i_{l}} .
$$

Note that any basis $\left\{e_{1}, \ldots, e_{n}\right\}$ in $\mathcal{H}$ induces a basis $\left\{e_{i_{1}} \otimes e_{i_{2}} \otimes \cdots \otimes e_{i_{k}} \mid i_{1}, \ldots, i_{k} \in\{1, \ldots, n\}\right\}$ in $\mathcal{H}^{\otimes k}$. Therefore, any $u \in \mathcal{H}^{\otimes k}$ can be uniquely written as a linear combination

$$
u=\sum_{i_{1}, \ldots, i_{k}=1}^{n} u^{i_{1} \ldots i_{k}} e_{i_{1}} \otimes \ldots \otimes e_{i_{k}} .
$$


If $u \in \mathcal{H}^{\vee k}$, then the tensor coefficients $u^{i_{1} \ldots i_{k}}$ are totally symmetric and, after applying the symmetrization projection to (91), we get

$$
u=\sum_{i_{1}, \ldots, i_{k}=1}^{n} u^{i_{1} \ldots i_{k}} e_{i_{1}} \vee \ldots \vee e_{i_{k}}
$$

Similarly, if $u \in \mathcal{H}^{\wedge k}$, the tensor coefficients $u^{i_{1} \ldots i_{k}}$ are totally antisymmetric and

$$
u=\sum_{i_{1}, \ldots, i_{k}=1}^{n} u^{i_{1} \ldots i_{k}} e_{i_{1}} \wedge \ldots \wedge e_{i_{k}}
$$

We will refer to the coefficients $u^{i_{1} \ldots i_{k}}$ as to the coefficients of $u$ in the basis $\left\{e_{1}, \ldots, e_{n}\right\}$.

\section{Tensor duality and contractions}

Starting with the canonical duality (pairing) $\langle$,$\rangle between vectors from \mathcal{H}$ and covectors from $\mathcal{H}^{*}$, with an obvious prolongation to a paring between $\mathcal{H}^{\otimes k}$ and $\left(\mathcal{H}^{*}\right)^{\otimes k}$,

$$
\left\langle f_{1} \otimes \cdots \otimes f_{k}, g_{1} \otimes \cdots \otimes g_{k}\right\rangle=\prod_{i=1}^{k}\left\langle f_{i}, g_{i}\right\rangle,
$$

and viewing symmetric and antisymmetric tensors as canonically embedded in the tensor algebra, we find canonical pairings between $\mathcal{H}^{\vee k}$ and $\left(\mathcal{H}^{*}\right)^{\vee k}$, as well as between $\mathcal{H}^{\wedge k}$ and $\left(\mathcal{H}^{*}\right)^{\wedge k}$. For $f_{1}, \ldots, f_{k} \in \mathcal{H}$ and $g_{1}, \ldots, g_{k} \in \mathcal{H}^{*}$, we get

$$
\left\langle f_{1} \vee \cdots \vee f_{k}, g_{1} \vee \cdots \vee g_{k}\right\rangle=\frac{1}{(k !)^{2}} \sum_{\sigma, \tau \in S_{k}} \prod_{i=1}^{k}\left\langle f_{\sigma(i)}, g_{\tau(i)}\right\rangle=\frac{1}{k !} \operatorname{per}\left(\left\langle f_{i}, g_{j}\right\rangle\right) .
$$

Here, $\sum_{\tau \in S_{k}} \prod_{i=1}^{k} a_{i \tau(i)}=\operatorname{per}\left(a_{i j}\right)$ is the permanent of the matrix $A=\left(a_{i j}\right)$. Similarly,

$$
\left\langle f_{1} \wedge \cdots \wedge f_{k}, g_{1} \wedge \cdots \wedge g_{k}\right\rangle=\frac{1}{k !} \operatorname{det}\left(\left\langle f_{i}, g_{j}\right\rangle\right) .
$$

Quite similarly one can prove that the natural Hermitian product on $\mathcal{H}^{\otimes}$, for which the grading is the decomposition into orthogonal subspaces and

$$
\left\langle f_{1} \otimes \cdots \otimes f_{k} \mid f_{1}^{\prime} \otimes \cdots \otimes f_{k}^{\prime}\right\rangle=\prod_{i=1}^{k}\left\langle f_{i} \mid f_{i}^{\prime}\right\rangle
$$

induces Hermitian products on the subspaces $\mathcal{H}^{\vee k}$ and $\mathcal{H}^{\wedge k}$ which read, respectively,

$$
\begin{aligned}
\left\langle f_{1} \vee \cdots \vee f_{k} \mid f_{1}^{\prime} \vee \cdots \vee f_{k}^{\prime}\right\rangle & =\frac{1}{k !} \operatorname{per}\left(\left\langle f_{i} \mid f_{j}^{\prime}\right\rangle\right), \\
\left\langle f_{1} \wedge \cdots \wedge f_{k} \mid f_{1}^{\prime} \wedge \cdots \wedge f_{k}^{\prime}\right\rangle & =\frac{1}{k !} \operatorname{det}\left(\left\langle f_{i} \mid f_{j}^{\prime}\right\rangle\right) .
\end{aligned}
$$

Given a basis $f_{1}, \ldots, f_{n}$ of $\mathcal{H}$ and the dual basis $f_{1}^{*}, \ldots, f_{n}^{*}$ of $\mathcal{H}^{*}$, we have the induced bases:

$$
f_{1}^{k_{1}} \vee \cdots \vee f_{n}^{k_{n}}, \quad k_{1}+\cdots+k_{n}=k
$$


of $\mathcal{H}^{\vee k}$, and

$$
f_{i_{1}} \wedge \cdots \wedge f_{i_{k}}, \quad 1 \leq i_{1}<i_{2}<\cdots<i_{k} \leq n
$$

of $\mathcal{H}^{\wedge k}$. The dual bases read

$$
\frac{k !}{k_{1} ! \cdots k_{n} !}\left(f_{1}^{*}\right)^{k_{1}} \vee \cdots \vee\left(f_{n}^{*}\right)^{k_{n}}, \quad k_{1}+\cdots+k_{n}=k,
$$

and

$$
k ! f_{i_{1}}^{*} \wedge \cdots \wedge f_{i_{k}}^{*}, \quad 1 \leq i_{1}<i_{2}<\cdots<i_{k} \leq n .
$$

Analogously, any orthonormal basis $e_{1}, \ldots, e_{n}$ of $\mathcal{H}$ induces canonical orthonormal bases

$$
\sqrt{\frac{k !}{k_{1} ! \cdots k_{n} !}} e_{1}^{k_{1}} \vee \cdots \vee e_{n}^{k_{n}}, \quad k_{1}+\cdots+k_{n}=k
$$

of $\mathcal{H}^{\vee k}$, and

$$
\sqrt{k !} e_{i_{1}} \wedge \cdots \wedge e_{i_{k}}, \quad 1 \leq i_{1}<i_{2}<\cdots<i_{k} \leq n
$$

of $\mathcal{H}^{\wedge k}$.

The canonical pairings between $\mathcal{H}^{\vee k}$ and $\left(\mathcal{H}^{*}\right)^{\vee k}$ on one hand, and $\mathcal{H}^{\wedge k}$ and $\left(\mathcal{H}^{*}\right)^{\wedge k}$ on the other, can be generalized to certain 'pairings' (contractions or inner products) between $\mathcal{H}^{\vee k}$ and $\left(\mathcal{H}^{*}\right)^{\vee l}$ on one hand, and $\mathcal{H}^{\wedge k}$ and $\left(\mathcal{H}^{*}\right)^{\wedge l}$ on the other. For the standard simple tensors $f=f_{1} \otimes \cdots \otimes f_{k} \in \mathcal{H}^{\otimes k}$ and $g=g_{1} \otimes \cdots \otimes g_{l} \in\left(\mathcal{H}^{*}\right)^{\otimes l}$, we just put

$$
\imath_{g} f=\left\langle f_{1} \otimes \cdots \otimes f_{l}, g_{1} \otimes \cdots \otimes g_{l}\right\rangle f_{l+1} \otimes \cdots \otimes f_{k}
$$

if $l \leq k$ and $\iota_{g} f=0$ if $l>k$, and extend it by linearity to all tensors. It is easy to see now that, if $v=f_{1} \vee \cdots \vee f_{k} \in \mathcal{H}^{\vee k} \subset \mathcal{H}^{\otimes k}$ and $\nu=g_{1} \vee \cdots \vee g_{l} \in\left(\mathcal{H}^{*}\right)^{\vee l} \subset\left(\mathcal{H}^{*}\right)^{\otimes l}$ then $\imath_{\nu} v \in \mathcal{H}^{\vee(k-l)}$.

Similarly, $\iota_{\omega} w \in \mathcal{H}^{\wedge(k-l)}$, if $w \in \mathcal{H}^{\wedge k} \subset \mathcal{H}^{\otimes k}$ and $\omega \in\left(\mathcal{H}^{*}\right)^{\wedge l} \subset\left(\mathcal{H}^{*}\right)^{\otimes l}$. Explicitly,

$$
\begin{array}{r}
\imath_{g_{1} \vee \cdots \vee g_{l}} f_{1} \vee \cdots \vee f_{k}=\frac{1}{k ! l !} \sum_{\substack{\sigma \in S_{k} \\
\tau \in S_{l}}} \prod_{j=1}^{l}\left\langle f_{\sigma(j)}, g_{\tau(j)}\right\rangle f_{\sigma(l+1)} \otimes \cdots \otimes f_{\sigma(k)} \\
=\frac{(k-l) !}{k !} \sum_{\substack{S \in S(l, k-l) \\
\tau \in S_{l}}} \prod_{j=1}^{l}\left\langle f_{S(j)}, g_{\tau(j)}\right\rangle f_{S(l+1)} \vee \cdots \vee f_{S(k)},
\end{array}
$$

where $S(l, k-l)$ denotes the set of all $(l, k-l)$ shuffles.

For skew-symmetric tensors,

$$
\begin{aligned}
\imath_{g_{1} \wedge \cdots \wedge g_{l}} f_{1} \wedge \cdots \wedge f_{k}=\frac{1}{k ! l !} \sum_{\substack{\sigma \in S_{k} \\
\tau \in S_{l}}}(-1)^{\sigma}(-1)^{\tau} \prod_{j=1}^{l}\left\langle f_{\sigma(j)}, g_{\tau(j)}\right\rangle f_{\sigma(l+1)} \otimes \cdots \otimes f_{\sigma(k)} \\
=\frac{(k-l) !}{k !} \sum_{\substack{S \in S(l, k-l) \\
\tau \in S_{l}}}(-1)^{\sigma}(-1)^{\tau} \prod_{j=1}^{l}\left\langle f_{S(j)}, g_{\tau(j)}\right\rangle f_{S(l+1)} \wedge \cdots \wedge f_{S(k)} .
\end{aligned}
$$

In particular,

$$
\imath_{g_{1} \vee \cdots \vee g_{k}} f_{1} \vee \cdots \vee f_{k}=\left\langle f_{1} \vee \cdots \vee f_{k}, g_{1} \vee \cdots \vee g_{k}\right\rangle=\frac{1}{k !} \operatorname{per}\left(\left\langle f_{i} \mid g_{j}\right\rangle\right),
$$


and

$$
\imath_{g_{1} \wedge \cdots \wedge g_{k}} f_{1} \wedge \cdots \wedge f_{k}=\left\langle f_{1} \wedge \cdots \wedge f_{k}, g_{1} \wedge \cdots \wedge g_{k}\right\rangle=\frac{1}{k !} \operatorname{det}\left(\left\langle f_{i} \mid g_{j}\right\rangle\right) .
$$

Moreover,

$$
\begin{aligned}
\imath_{g_{1} \vee \cdots \vee g_{k-1}} f_{1} \vee \cdots \vee f_{k} & =\frac{1}{k} \sum_{j=1}^{k}\left\langle f_{1} \vee \stackrel{v}{\vee} \vee \vee f_{k}, g_{1} \vee \cdots \vee g_{k-1}\right\rangle f_{j} \\
& =\frac{1}{k !} \sum_{j=1}^{k} \operatorname{per}\left(\left\langle f_{i} \mid g_{s}\right\rangle_{i \neq j}\right) f_{j}
\end{aligned}
$$

and

$$
\begin{aligned}
\imath_{g_{1} \wedge \cdots \wedge g_{k-1}} f_{1} \wedge \cdots \wedge f_{k} & =\frac{1}{k} \sum_{j=1}^{k}(-1)^{k-j}\left\langle f_{1} \wedge \stackrel{\sim}{\vee} \wedge \wedge f_{k}, g_{1} \wedge \cdots \wedge g_{k-1}\right\rangle f_{j} \\
& =\frac{1}{k !} \sum_{j=1}^{k}(-1)^{k-j} \operatorname{det}\left(\left\langle f_{i} \mid g_{s}\right\rangle_{i \neq j}\right) f_{j}
\end{aligned}
$$

\section{The S-rank}

There are many concepts of a rank of a tensor used to describe its complexity. One of the simplest and most natural is the one based on the inner product operators defined in the previous section. We will call it the $S$-rank, since it turns out to be a natural generalization of the Schmidt rank of 2-tensors.

Definition 4.1. Let $u \in \mathcal{H}^{\otimes k}$. By the $S$-rank of $u$, we understand the maximum of dimensions of the linear spaces $\imath_{\mathcal{H}}^{k-1} \sigma(u)$, for $\sigma \in S_{k}$, which are the images of the contraction maps

$$
\left(\mathcal{H}^{*}\right)^{\otimes(k-1)} \ni \mu \mapsto \imath_{\mu} \sigma(u) \in \mathcal{H} .
$$

Theorem 4.1. If $u \in \mathcal{H}^{\vee k}$ (resp., $u \in \mathcal{H}^{\wedge k}$ ), then the S-rank of $u$ equals the dimension of the linear space being the image of the contraction map

$$
\left(\mathcal{H}^{*}\right)^{\vee(k-1)} \ni \mu \mapsto \imath_{\mu} u \in \mathcal{H},
$$

(resp.,

$$
\left.\left(\mathcal{H}^{*}\right)^{\wedge(k-1)} \ni \mu \mapsto \imath_{\mu} u \in \mathcal{H}\right) .
$$

Proof: It follows immediately from the observation that a contraction of a symmetric (res., antisymmetric) tensor $u$ with a tensor $\mu \in\left(\mathcal{H}^{*}\right)^{\otimes i}$ is the same as its contraction with the symmetrization (resp., antisymmetrization) of $\mu, \imath_{\mu} u=\imath_{\pi^{\vee}(\mu)} u,\left(\right.$ resp. $\left.\imath_{\mu} u=\imath_{\pi^{\wedge}(\mu)} u\right)$ and that, for $\sigma \in S_{k}, \sigma(u)= \pm u$.

Example 4.1. Let $e_{1}, e_{2}$ be orthogonal vectors in $\mathcal{H}$. The vector

$$
u=e_{1} \vee e_{2}=\frac{1}{2}\left(e_{1} \otimes e_{2}+e_{2} \otimes e_{1}\right)
$$

has S-rank 2. Indeed, for each $x \in \mathcal{H}$,

$$
\imath_{x} u=\frac{1}{2} \imath_{x}\left(e_{1} \otimes e_{2}+e_{2} \otimes e_{1}\right)=\frac{1}{2}\left(\left\langle x \mid e_{1}\right\rangle e_{2}+\left\langle x \mid e_{2}\right\rangle e_{1}\right),
$$

so that $\imath_{\mathcal{H}} u$ is spanned by $\left\{e_{1}, e_{2}\right\}$. 
Example 4.2. Let us take $\mathcal{H}$ of dimension 3 and an orthonormal basis $e_{i}, i=1,2,3$. Let $u \in \mathcal{H}^{\vee 4}$ in the polynomial notation reads

$$
\begin{aligned}
u= & e_{1}^{4}+e_{2}^{4}+16 e_{3}^{4}+4 e_{1}^{3} e_{2}+8 e_{1}^{3} e_{3}+4 e_{1} e_{2}^{3}+8 e_{2}^{3} e_{3}+32 e_{1} e_{3}^{3}+32 e_{2} e_{3}^{3} \\
& +6 e_{1}^{2} e_{2}^{2}+24 e_{1}^{2} e_{3}^{2}+24 e_{2}^{2} e_{3}^{2}+24 e_{1}^{2} e_{2} e_{3}+24 e_{1} e_{2}^{2} e_{3}+48 e_{1} e_{2} e_{3}^{2} .
\end{aligned}
$$

The vectors

$$
e_{1}^{3}, e_{2}^{3}, e_{3}^{3}, e_{1}^{2} e_{2}, e_{1}^{2} e_{3}, e_{1} e_{2}^{2}, e_{2}^{2} e_{3}, e_{1} e_{3}^{2}, e_{2} e_{3}^{2}, e_{1} e_{2} e_{3}
$$

form a basis of $\mathcal{H}^{\vee 3}$ and it can be directly checked with the use of (26) that any of these vectors, say $\mu$, contracted with $u$ gives a vector proportional to $e_{1}+e_{2}+2 e_{3}$. For instance, the only non-zero parts of $\imath_{\mu} u$ for $\mu=e_{2}^{3}$ are

$$
\imath_{e_{2}^{3}}(u)=\imath_{e_{2}^{3}}\left(e_{2}^{4}+4 e_{1} e_{2}^{3}+8 e_{2}^{3} e_{3}\right)=e_{2}+e_{1}+2 e_{3}
$$

and

$$
{ }^{\imath} e_{1}^{2} e_{3}(u)=\imath_{e_{1}^{2} e_{3}}\left(8 e_{1}^{3} e_{3}+24 e_{1}^{2} e_{3}^{2}+24 e_{1}^{2} e_{2} e_{3}\right)=\left\|e_{1}^{2} e_{3}\right\|^{2}\left(6 e_{1}+12 e_{3}+6 e_{2}\right)=2\left(e_{1}+2 e_{3}+e_{2}\right) .
$$

It follows that $\imath_{\mathcal{H}} \vee 3(u)$ is 1 -dimensional, so the S-rank of $u$ is 1 .

Example 4.3. For $\mathcal{H}$ with the basis as above, consider $w \in \mathcal{H}^{\wedge 2}$ of the form

$$
w=e_{1} \wedge e_{2}+e_{2} \wedge e_{3} .
$$

Let us see that the S-rank of $w$ is 2. Indeed,

$$
\imath_{e_{1}} w=\frac{1}{2} e_{2}, \quad \imath_{e_{2}} w=\frac{1}{2}\left(e_{3}-e_{1}\right), \quad \imath_{e_{3}} w=-\frac{1}{2} e_{2},
$$

so that $\imath_{\mathcal{H}} w$ is spanned by $e_{2}$ and $e_{3}-e_{1}$.

Example 4.4. For $\mathcal{H}$ with an orthonormal basis $e_{1}, e_{2}, e_{3}, e_{4}$ the tensor

$$
w=e_{1} \wedge e_{2}+e_{3} \wedge e_{4}
$$

has the S-rank 4. Indeed,

$$
\imath_{e_{1}} w=\frac{1}{2} e_{2}, \quad \imath_{e_{2}} w=-\frac{1}{2} e_{1}, \quad \imath_{e_{3}} w \frac{1}{2} e_{4}, \quad \imath_{e_{4}} w=-\frac{1}{2} e_{3} .
$$

Theorem 4.2. (a) The minimal possible $S$-rank of a non-zero tensor $u \in \mathcal{H}^{\otimes k}$ equals 1 . A tensor $u \in \mathcal{H}^{\otimes k}$ is of S-rank 1 if and only if $u$ is decomposable, i.e., $u$ can be written in the form

$$
u=f_{1} \otimes \cdots \otimes f_{k}, \quad f_{i} \in \mathcal{H}, \quad f_{i} \neq 0 .
$$

Such tensors span $\mathcal{H}^{\otimes k}$.

(b) The minimal possible $S$-rank of a non-zero tensor $v \in \mathcal{H}^{\vee k}$ equals 1 . A tensor $v \in \mathcal{H}^{\vee k}$ is of S-rank 1 if and only if $v$ can be written in the form

$$
v=f \vee \cdots \vee f, \quad f \in \mathcal{H}, \quad f \neq 0 .
$$

Such tensors span $\mathcal{H}^{\vee k}$. 
(c) The minimal possible S-rank of a non-zero tensor $w \in \mathcal{H}^{\wedge k}$ equals $k$. A tensor $w \in \mathcal{H}^{\wedge k}$ is of S-rank $k$ if and only if $w$ can be written in the form

$$
w=f_{1} \wedge \cdots \wedge f_{k},
$$

where $f_{1}, \ldots, f_{k} \in \mathcal{H}$ are linearly independent. Such tensors span $\mathcal{H}^{\wedge k}$.

Proof: The 'if' parts of the above statements are obvious, so we shall prove 'only if'. If the dimension of $\imath_{\mathcal{H}}^{k-1} u$ is 1 , thus the space is spanned by some $f=f_{k} \in \mathcal{H}$, then clearly $u=u^{\prime} \otimes f$ for some $u^{\prime} \in \mathcal{H}^{\otimes(k-1)}$. If, in turn, the tensor is symmetric, then clearly $u$ is proportional to $f \otimes \cdots \otimes f$. In the general case we get a similar fact for $\sigma(u)$ with $\sigma \in S_{k}$, so $u=f_{1} \otimes \cdots \otimes f_{k}$. If $u$ is skew-symmetric and $f_{1}, \ldots, f_{r}$ span $\imath_{\mathcal{H}}^{k-1} u$, then $u$ is a linear combination of tensor products of these vectors, so of $f_{i_{1}} \wedge \cdots \wedge f_{i_{k}}$. Hence, $r \geq k$, and $r=k$ if and only if $u$ is proportional to $f_{1} \wedge \cdots \wedge f_{k}$. That simple tensors span the corresponding spaces is pretty well known for general and antisymmetric tensors. For symmetric tensors it follows from the fact that powers of linear functions span the spaces of polynomials with coefficients in a field of characteristic 0 .

Definition 4.2. Tensors of minimal S-rank in $\mathcal{H}^{\otimes k}$ (resp., $\mathcal{H}^{\vee k}, \mathcal{H}^{\wedge k}$ ) we will call simple (resp., simple symmetric, simple antisymmetric).

Theorem 4.2 immediately implies the following.

Corollary 4.1. The S-rank is 1 for simple and simple symmetric tensors, and it is $k$ for simple antisymmetric tensors from $\mathcal{H}^{\wedge k}$. Simple tensor have the form (35), simple symmetric tensors have the form (36), and simple antisymmetric tensor have the form (37).

Example 4.5. The symmetric tensor $u$ defined in (32), Example 4.2, has the S-rank 1, so it is simple. As a matter of fact,

$$
u=\left(e_{1}+e_{2}+2 e_{3}\right)^{4} .
$$

Also the antisymmetric tensor $w$ defined in (33), Example 4.3, is simple:

$$
w=e_{2} \wedge\left(e_{3}-e_{1}\right) .
$$

Remark 4.1. Of course, for distinguishable particles there is no need to use the same Hilbert space $\mathcal{H}$ in the tensor products. We can use the tensor product $\mathcal{H}_{1} \otimes \cdots \otimes \mathcal{H}_{k}$ instead, with simple tensors being decomposable: $u=f_{1} \otimes \cdots \otimes f_{k}, f_{i} \in \mathcal{H}_{i}$.

\section{Various characterizations of simple tensors}

For $\mathcal{H}_{0}^{\otimes 2}$, where $\mathcal{H}_{0}=\mathcal{H}_{1} \otimes \cdots \otimes \mathcal{H}_{k}$, we denote with $\sigma_{i}: \mathcal{H}_{0}^{\otimes 2} \rightarrow \mathcal{H}_{0}^{\otimes 2}, i=1, \ldots, k$, the transposition with respect to the $i$-th and the $(k+i)$-th factor,

$$
\begin{aligned}
& \sigma_{i}\left(a_{1} \otimes \cdots \otimes a_{2 k}\right)= \\
& a_{1} \otimes \cdots \otimes a_{i-1} \otimes a_{k+i} \otimes a_{i+1} \otimes \cdots \otimes a_{k+i-1} \otimes a_{i} \otimes a_{k+i+1} \otimes \cdots \otimes a_{2 k},
\end{aligned}
$$

and with $\tau_{k}: \mathcal{H}_{0}^{\otimes 2} \rightarrow \mathcal{H}_{0}^{\otimes 2}$ - the cyclic permutation that moves the last factor into the first place:

$$
\tau_{k}\left(a_{1} \otimes \cdots \otimes a_{2 k}\right)=a_{2 k} \otimes a_{1} \otimes \cdots \otimes a_{2 k-1} .
$$


Theorem 5.1. For $u \in \mathcal{H}_{1} \otimes \cdots \otimes \mathcal{H}_{k}$ the following are equivalent:

(a) $u$ is simple;

(b) $\forall \sigma \in S_{k} \forall \mu_{1}, \mu_{2} \in \mathcal{H}_{\sigma(1)}^{*} \otimes \cdots \otimes \mathcal{H}_{\sigma(k-1)}^{*} \quad \imath_{\mu_{1}} \sigma(u) \wedge \imath_{\mu_{2}} \sigma(u)=0$,

(c) $\sigma_{i}(u \otimes u)=u \otimes u$ for $i=1, \ldots, k$.

Proof: (a) $\Rightarrow$ (b) is obvious in view of Theorem 4.2 (a). Also (b) $\Rightarrow$ (c) is clear, so assume $\sigma_{k}(u \otimes u)=u \otimes u$. This implies that the dimension of the space $\imath_{\mathcal{H}}^{k-1} u$ is 1 . Indeed, if this space is spanned by linearly independent vectors $g_{1}, \ldots, g_{r}$, then $u=\sum_{j=1}^{r} u_{r} \otimes g_{r}$ for some linearly independent $u_{j} \in \mathcal{H}^{\otimes(k-1)}$. Since $\sigma_{k}(u \otimes u)=u \otimes u$ means that

$$
\sum_{j, s=1}^{r} u_{j} \otimes g_{j} \otimes u_{s} \otimes g_{s}=\sum_{j, s=1}^{r} u_{j} \otimes g_{s} \otimes u_{s} \otimes g_{j},
$$

we conclude that $r=1$, so $u=u^{\prime} \otimes f_{k}$ for some $f_{k} \in \mathcal{H}$ and $u^{\prime} \in \mathcal{H}^{\otimes(k-1)}$. A similar reasoning, applied to the identities $\sigma_{i}(u \otimes u)=u \otimes u$ with $i=1, \ldots, k-1$, implies that $u=f_{1} \otimes \cdots \otimes f_{k}$.

Theorem 5.2. For a symmetric tensor $v \in \mathcal{H}^{\vee k}$ the following are equivalent

(a) $v$ is simple symmetric,

(b) $\forall \nu_{1}, \nu_{2} \in\left(\mathcal{H}^{*}\right)^{\vee(k-1)} \imath_{\nu_{1}} v \wedge \imath_{\nu_{2}} v=0$,

(c) $v \otimes v=\sigma_{k}(v \otimes v)$.

Proof: (a) $\Rightarrow(\mathrm{b})$ is obvious in view of Theorem 4.2 (b). As (b) implies $v=v^{\prime} \otimes f_{k}$ for some $f_{k} \in \mathcal{H}$, also (b) $\Rightarrow$ (c) is clear. The condition (c), in turn, for symmetric tensor yields $v \otimes v=\sigma_{i}(v \otimes v)$ for all $i=1, \ldots, k$, so $v=f_{1} \otimes \cdots \otimes f_{k}$ as above, thus $v=f \otimes \cdots \otimes f$ by symmetry.

Theorem 5.3. For an antisymmetric tensor $w \in \mathcal{H}^{\wedge k}$ the following are equivalent

(a) $w$ is simple antisymmetric,

(b) $\forall \omega \in\left(\mathcal{H}^{*}\right)^{\wedge(k-1)} w \wedge \imath_{\omega} w=0$,

(c) $\left(\pi_{k+1}^{\wedge} \otimes i d_{\mathcal{H}^{\otimes(k-1)}}\right)\left(\tau_{k}(w \otimes w)\right)=0$.

Proof: $(\mathrm{a}) \Rightarrow(\mathrm{b})$ is obvious in view of Theorem 4.2 (c). Also (b) $\Rightarrow(\mathrm{a})$ is clear and well known. We shall show that $(b)$ and $(c)$ are equivalent.

Let us write $w$ as a sum of simple antisymmetric tensors, $w=\sum_{j=1}^{m} w_{j}$, with minimal $m$. Write $w_{j}=f_{j}^{1} \wedge \cdots \wedge f_{j}^{k}$ and denote $w_{j}^{s}=f_{j}^{1} \wedge \cdots \widehat{f_{j}^{s}} \wedge \cdots \wedge f_{j}^{k}, s=1, \ldots, k$. Here, "^" stands for the omission. As the number of simple tensors is minimal, the tensors $w_{j}^{s}$ are linearly independent in $\mathcal{H}^{\wedge(k-1)}$.

Observe now that

$$
w_{j}=\sum_{s} \frac{(-1)^{k-s}}{k} w_{j}^{s} \otimes f_{j}^{s}
$$


so that

$$
\left(\pi_{k+1}^{\wedge} \otimes i d_{\mathcal{H} \otimes(k-1)}\right)\left(\tau_{k}(w \otimes w)\right)=\sum_{j, s} \frac{(-1)^{k-s}}{k} f_{j}^{s} \wedge w \otimes w_{j}^{s} .
$$

Since $w_{j}^{s}$ are linearly independent, the latter vanishes if and only if all $f_{j}^{s} \wedge w$ vanish, that is clearly equivalent to (b).

Remark 5.1. The conditions (c) in Theorems 5.1, 5.2, and 5.3 have formally this advantage over the corresponding conditions (b) that they are directly verifiable, as they do not contain general quantifiers referring to infinite sets.

Note that all we have said remains valid for an arbitrary vector space over a field of characteristics 0 . The Hermitian structure played no role yet.

Let us note first that the S-rank is associated with a point in a projective space rather than with a tensor itself. Hence, we can restrict considerations to tensors of length 1 (as vectors in the Hilbert space $\mathcal{H}_{1} \otimes \cdots \otimes \mathcal{H}_{k}$ ). According to Theorem 5.1, a tensor $u \in \mathcal{H}_{1} \otimes \cdots \otimes \mathcal{H}_{k}$ is simple if and only if $u \otimes u=\sigma_{i}(u \otimes u)$ for all $i=1, \ldots, k$, where $\sigma_{i}$ interweaves the two copies of $\mathcal{H}_{i}$ in $\mathcal{H}_{1} \otimes \cdots \otimes \mathcal{H}_{k} \otimes \mathcal{H}_{1} \otimes \cdots \otimes \mathcal{H}_{k}$. Note that $\sigma_{i}$ acts as a unitary operator in $\left(\mathcal{H}_{1} \otimes \cdots \otimes \mathcal{H}_{k}\right)^{\otimes 2}$. This means, in turn, that

$$
\sum_{i=1}^{k}\left\|u \otimes u-\sigma_{i}(u \otimes u)\right\|^{2}=0
$$

The latter we can write as

$$
\sum_{i=1}^{k}\left\langle u \otimes u-\sigma_{i}(u \otimes u) \mid u \otimes u-\sigma_{i}(u \otimes u)\right\rangle=0,
$$

which, for tensors of length 1 is equivalent to $\sum_{i=1}^{k} \operatorname{Re}\left\langle u \otimes u \mid \sigma_{i}(u \otimes u)\right\rangle=k$, or, finally, to

$$
\operatorname{Re}\left\langle u \otimes u \mid\left(\sum_{i=1}^{k} \sigma_{i}\right)(u \otimes u)\right\rangle=k
$$

The Schwarz inequality yields now $u \otimes u=\bar{\sigma}(u \otimes u)$, where $\bar{\sigma}=\frac{1}{k} \sum_{i=1}^{n} \sigma_{i}$, i.e.,

$$
\bar{\sigma}\left(x_{1} \otimes \cdots \otimes x_{k} \otimes y_{1} \otimes \cdots \otimes y_{k}\right)=\frac{1}{k} \sum_{i=1}^{n} x_{1} \otimes \cdots \otimes y_{i} \otimes \cdots x_{k} \otimes y_{1} \otimes \cdots \otimes x_{i} \otimes \cdots \otimes y_{k} .
$$

In this way we have proven the following.

Theorem 5.4. Let $u \in \mathcal{H}_{1} \otimes \cdots \otimes \mathcal{H}_{k},\|u\|=1$. Then, $u$ is simple if and only if

$$
\langle u \otimes u, \bar{\sigma}(u \otimes u)\rangle=1 .
$$

For symmetric tensors, a similar fact can be proven analogously.

Theorem 5.5. Let $u \in \mathcal{H}^{\vee k},\|u\|=1$. Then, $u$ is simple symmetric if and only if

$$
\left\langle u \otimes u, \sigma_{k}(u \otimes u)\right\rangle=1 .
$$


The structure of a Hilbert space $\mathcal{H}$ has been used in [4, 5, 6] to define simple symmetric and simple antisymmetric tensors for tensors of order 2 by means of the Slater decomposition. Both decompositions are direct consequences of a possibility to write a complex symmetric (antisymmetric) matrix in a diagonal (block-diagonal) form by a unitary change of basis (Takagi theorem [16]). The existence of the Slater decompositions means that any symmetric tensor $v \in \mathcal{H} \vee \mathcal{H}$ and any antisymmetric tensor $w \in \mathcal{H} \wedge \mathcal{H}$ can be written as

$$
\begin{aligned}
v & =\sum_{i=1}^{n} \lambda_{i} e_{i} \vee e_{i}, \quad \lambda_{i}>0, \\
w & =\sum_{i=1}^{n} \lambda_{i} f_{i} \wedge f_{n+i}, \quad \lambda_{i}>0,
\end{aligned}
$$

for some orthonormal systems $\left(e_{i}\right)$ and $\left(f_{i}\right)$ of $\mathcal{H}$. The Slater rank is the number $n$ of terms in these decompositions. The above are clearly symmetric and antisymmetric analogues of the Schmidt decomposition: any tensor $u \in \mathcal{H}_{1} \otimes \mathcal{H}_{2}$ can be written in the form

$$
u=\sum_{i=1}^{n} \lambda_{i} e_{i} \otimes f_{i}, \quad \lambda_{i}>0,
$$

for some orthonormal systems: $\left(e_{i}\right)$ in $\mathcal{H}_{1}$, and $\left(f_{i}\right)$ in $\mathcal{H}_{2}$.

Theorem 5.6. For any 2-tensor $u \in \mathcal{H}_{1} \otimes \mathcal{H}_{2}$ its Schmidt rank equals its S-rank.

Proof: It is clear that the Schmidt decomposition (43) implies that the S-rank of $u$ is $n$. Conversely, if the S-rank of $u$ is $n$, then $u$ can be written in the form (43) with the only difference that the systems $\left(e_{i}\right)$ and $\left(f_{i}\right)$ are merely linearly independent. But this is a standard procedure, used in the proof of existence of the Schmidt decomposition, that we can choose $\left(e_{i}\right)$ and $\left(f_{i}\right)$ orthonormal.

Hence, the 2-tensors are simple (resp., simple symmetric, simple antisymmetric), if there exists a Schmidt (resp. Slater) decomposition with a single $\lambda_{i}>0$, i.e. they have the Schmidt (Slater) rank 1. Unfortunately, there are no direct analogues of these decompositions for tensors of higher orders.

On the other hand, as we have already seen, such type of a decomposition is not necessary to define (and check) which tensors are simple symmetric (resp., simple antisymmetric), as the S-rank can serve in these cases.

\section{$6 \quad$ Entanglement for multipartite Bose and Fermi systems}

Using the concept of simple tensors we can define simple (non-entangled or separable) and entangled pure states for multipartite systems of bosons and fermions.

\section{Definition 6.1.}

(a) A pure state $\rho_{v}$ on $\mathcal{H}^{\vee k}$ (resp., on $\mathcal{H}^{\wedge k}$ ), $\rho_{v}=\frac{|v\rangle\langle v|}{\|v\|^{2}}$, with $v \in \mathcal{H}^{\vee k}$ (resp., $v \in \mathcal{H}^{\wedge k}$ ), $v \neq 0$, is called a bosonic (resp., fermionic) simple (or non-entangled) pure state, if $v$ is a simple symmetric (resp., antisymmetric) tensor. If $v$ is not simple symmetric (resp., antisymmetric), we call $\rho_{v}$ a bosonic (resp., fermionic) entangled state. 
(b) A mixed state $\rho$ on $\mathcal{H}^{\vee k}$ (resp., on $\mathcal{H}^{\wedge k}$ ) we call bosonic (resp., fermionic) simple (or non-entangled) mixed state, if it can be written as a convex combination of bosonic (resp., fermionic) simple pure states. In the other case $\rho$ is called bosonic (resp., fermionic) entangled mixed state.

According to Theorem 4.2, bosonic simple pure $k$-states are of the form

$$
|e \vee \cdots \vee e\rangle\langle e \vee \cdots \vee e|
$$

for some unit vector $e \in \mathcal{H}$, and fermionic simple pure $k$-states are of the form

$$
k !\left|e_{1} \wedge \cdots \wedge e_{k}\right\rangle\left\langle e_{1} \wedge \cdots \wedge e_{k}\right|
$$

for some orthonormal system $e_{1}, \ldots, e_{k}$ in $\mathcal{H}$.

Fixing a base in $\mathcal{H}$ results in defining coefficients $\left[u^{i_{1} \ldots i_{k}}\right]$ of $u \in \mathcal{H}^{\otimes k}$. Formulae characterizing simple tensors, thus simple pure states, can be written in forms of quadratic equations with respect to these coefficients as follows. The corresponding characterization of entangled pure states are obtained by negation of the latter.

Theorem 6.1. The pure state $\rho_{u}$, associated with a tensor $u=\left[u^{i_{1} \ldots i_{k}}\right] \in \mathcal{H}^{\otimes k}$, is entangled if and only if there exist $i_{1}, \ldots, i_{k}, j_{1}, \ldots, j_{k}$, and $s=1, \ldots, k$ such that

$$
u^{i_{1} \ldots i_{s} \ldots i_{k}} u^{j_{1} \ldots j_{s} \ldots j_{k}} \neq u^{i_{1} \ldots j_{s} \ldots i_{k}} u^{j_{1} \ldots i_{s} \ldots j_{k}} .
$$

Proof: The tensor $u \otimes u$ has coefficients $u^{i_{1} \ldots i_{k}} u^{j_{1} \ldots k_{k}}$, so Eq.(44) expresses the fact that $u \otimes u \neq$ $\sigma_{s}(u \otimes u)$, and thus Theorem 6.1 is a direct consequence of Theorem 5.1.

Theorem 6.2. The bosonic pure state $\rho_{v}$, associated with a symmetric tensor $v=\left[v^{i_{1} \ldots i_{k}}\right] \in$ $\mathcal{H}^{\vee k}$, is bosonic entangled if and only if there exist $i_{1}, \ldots, i_{k}, j_{1}, \ldots, j_{k}$, such that

$$
v^{i_{1} \ldots i_{k-1} i_{k}} v^{j_{1} \ldots j_{k-1} j_{k}} \neq v^{i_{1} \ldots i_{k-1} j_{k}} v^{j_{1} \ldots j_{k-1} i_{k}} .
$$

Proof: A direct consequence of Theorem 5.2 ,

Example 6.1. The bosonic pure state associated with the symmetric tensor $u$ defined in (31) is entangled, as we know it has the S-rank 2. To apply our characterization (45), note that the only non-zero coefficients are $u^{12}=u^{21}=\frac{1}{2}$. Hence,

$$
0=u^{11} u^{22} \neq u^{12} u^{21}=\frac{1}{4} .
$$

Example 6.2. The bosonic pure state associated with the symmetric tensor $u$ defined in (32) is non-entangled, as we know it has the S-rank 1. To apply our characterization (45), note that, as easily checked, $u^{i_{1} i_{2} i_{3} i_{4}}=2^{i(3)}$, where $i(3)$ is the number of 3 's in the sequence $i=\left(i_{1}, i_{2}, i_{3}, i_{4}\right)$. Now it is clear that

$$
u^{i_{1} i_{2} i_{3} i_{4}} u^{j_{1} j_{2} j_{3} j_{4}}=2^{i(3)+j(3)},
$$

where $i(3)+j(3)$ is the number of 3 's in the sequence $\left(i_{1}, i_{2}, i_{3}, i_{4}, j_{1}, j_{2}, j_{3}, j_{4}\right)$ which remains unchanged under permutations. In particular,

$$
u^{i_{1} i_{2} i_{3} i_{4}} u^{j_{1} j_{2} j_{3} j_{4}}=u^{i_{1} i_{2} i_{3} j_{4}} u^{j_{1} j_{2} j_{3} i_{4}} .
$$


Theorem 6.3. The fermionic pure state $\rho_{w}$, associated with an antisymmetric tensor $w=$ $\left[w^{i_{1} \ldots i_{k}}\right] \in \mathcal{H}^{\wedge k}$, is fermionic entangled if and only if there exist $i_{1}, \ldots, i_{k+1}, j_{1}, \ldots, j_{k-1}$ such that

$$
w^{\left[i_{1} \ldots i_{k}\right.} w^{\left.i_{k+1}\right] j_{1} \ldots j_{k-1}} \neq 0,
$$

where the left-hand side is the antisymmetrization of $w^{i_{1} \ldots i_{k}} w^{i_{k+1} j_{1} \ldots j_{k-1}}$ with respect to indices $i_{1}, \ldots, i_{k+1}$.

Proof: A direct consequence of Theorem 5.3 .

In view of the above characterizations, it is obvious that the sets of entangled (entangled bosonic, entangled fermionic) pure states are open: pure states sufficiently close to entangled ones are entangled.

Example 6.3. The fermionic pure state associated with the antisymmetric tensor $w$ defined in (33) is non-entangled, as we know its S-rank is 2. To apply our characterization (46), let us note that the only non-zero coefficients are $w^{12}=-w^{21}=w^{23}=-w^{32}=\frac{1}{2}$. The antisymmetrization $w^{\left[i_{1} i_{2}\right.} w^{\left.i_{3}\right] j}$ can be non-zero only if $\left(i_{1}, i_{2}, i_{3}\right)$ is a permutation of $(1,2,3)$. But, as easily seen,

$$
w^{[12} w^{3] j}=\frac{1}{3}\left(w^{12} w^{3 j}-w^{32} w^{1 j}\right)=0
$$

for $j=1,2,3$.

Example 6.4. The fermionic pure state associated with the antisymmetric tensor $w$ defined in (34) is entangled, as we know its S-rank is 4. To apply our characterization (46), let us note that the only non-zero coefficients are $w^{12}=-w^{21}=w^{34}=-w^{43}=\frac{1}{2}$. As easily seen,

$$
w^{[12} w^{3] 4}=\frac{1}{3} w^{12} w^{34}=\frac{1}{12} \neq 0 .
$$

\section{Jamiołkowski isomorphisms for bi-partite systems of bosons and fermions}

In the theory of entanglement there exists a useful tool for investigating, on one hand, entanglement properties of states and, on the other, structure of positivity-preserving and hermiticitypreserving maps between matrix algebras called the Jamiołkowski isomorphism [17]. Originally proposed in [18] as an instrument for checking the property of preserving positive semidefiniteness for a linear map between two matrix algebras in finite dimensional spaces, it was later used to prove the so-called operator form representation for linear maps on quantum states (i.e. positive semi-definite operators) [19]. The construction can be extended to the infinite-dimensional setting if the restriction to Hilbert-Schmidt operators is imposed [20]) which significantly heightens the usefulness of the Jamiołkowski isomorphism in cases when finite-dimensional description of q quantum system is not suitable. An in-depth description of the applicability the Jamiołkowski isomorphism is given in [21] where various properties of entanglement and separability of states are paralleled with features of the corresponding linear maps.

One of the most straightforward applications of the Jamiołkowski isomorphism is a characterization of separability of a state in terms of the rank of the corresponding map. In the following we show how this decription can be extended to states of indistinguishable particles. 
Let us recall (see e.g. [20]) that for two Hilbert spaces $\mathcal{H}_{1}$ and $\mathcal{H}_{2}$ we have the following diagram consisting of Jamiotkowski isomorphisms:

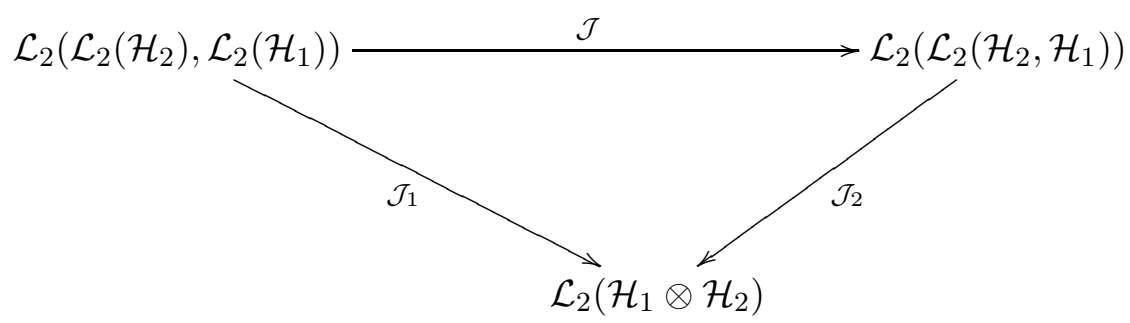

Here, with $\mathcal{L}_{2}\left(\mathcal{H}_{1}, \mathcal{H}_{2}\right)$ we denote the Hilbert space of Hilbert-Schmidt maps from $\mathcal{H}_{1}$ into $\mathcal{H}_{2}$. Of course, this space reduces to all complex linear maps, if $\mathcal{H}_{1}$ or $\mathcal{H}_{2}$ is finite-dimensional. Note that we write shorter $\mathcal{L}_{2}(\mathcal{H})$ for $\mathcal{L}_{2}(\mathcal{H}, \mathcal{H})$. Note also that according to the obvious identification $\mathcal{L}_{2}\left(\mathcal{H}_{2}, \mathcal{H}_{1}\right) \simeq \mathcal{H}_{1} \otimes \mathcal{H}_{2}^{*}$ and the identification

$$
\mathcal{L}\left(\mathcal{H}_{2}, \mathcal{H}_{1}\right)^{*} \simeq \mathcal{L}\left(\mathcal{H}_{1}, \mathcal{H}_{2}\right)
$$

induced by the natural pairing

$$
\mathcal{L}\left(\mathcal{H}_{2}, \mathcal{H}_{1}\right) \times \mathcal{L}\left(\mathcal{H}_{1}, \mathcal{H}_{2}\right) \ni(A, B) \mapsto \operatorname{Tr}(A \circ B) \in \mathbb{C},
$$

we can rewrite the above diagram in the form

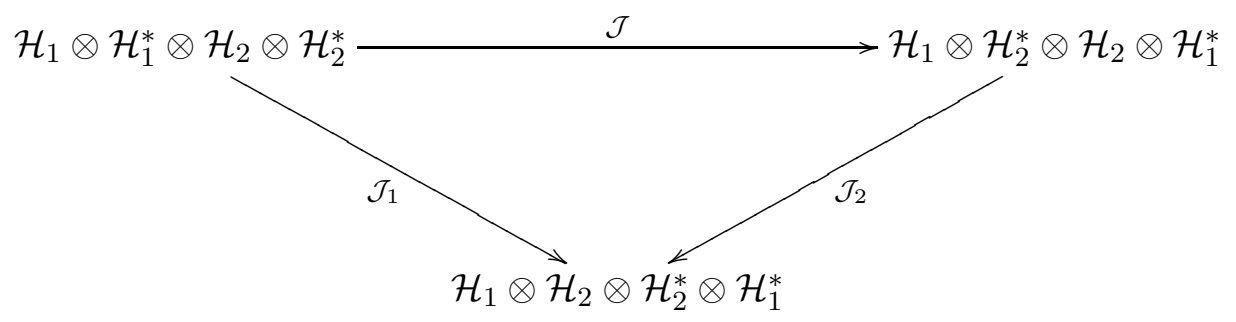

in which the Jamiołkowski isomorphisms reduce to appropriate permutations of tensors. In the case when $\mathcal{H}_{1}=\mathcal{H}_{2}$ the whole picture reduces to

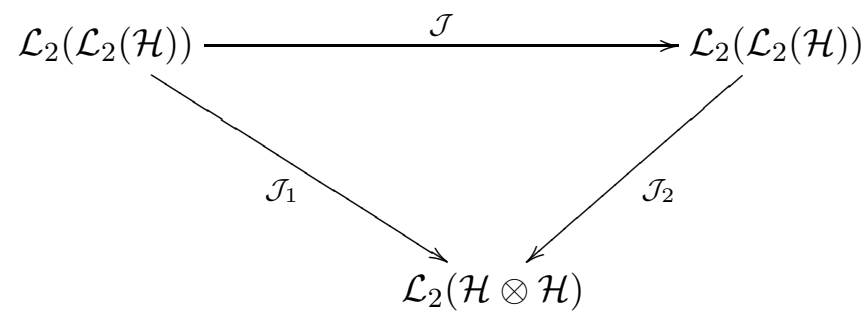

We can now decompose $\mathcal{H} \otimes \mathcal{H}$ into $(\mathcal{H} \vee \mathcal{H}) \oplus(\mathcal{H} \wedge \mathcal{H})$ and reduce our diagram to maps on each of these components. In this way we get

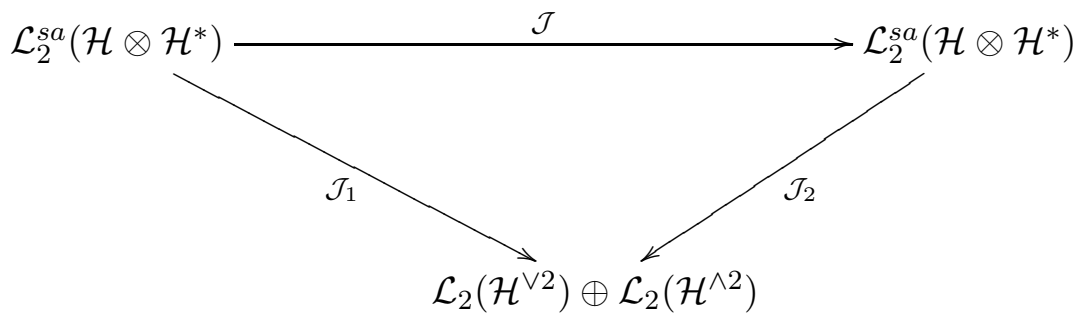


Here, $\mathcal{L}_{2}^{s a}\left(\mathcal{H} \otimes \mathcal{H}^{*}\right)$ is the space of self-adjoint Hilbert-Schmidt operators $\Phi, \Phi=\Phi^{*}$, on $\mathcal{H} \otimes \mathcal{H}^{*}=$ $\mathcal{L}_{2}(\mathcal{H})$ according to the identification $\mathcal{L}_{2}(\mathcal{H}) \simeq\left(\mathcal{L}_{2}(\mathcal{H})\right)^{*}$ (see (48)) related with the pairing (49). Note that this is different from hermicity, $\Phi^{*} \neq \Phi^{\dagger}$, since $\Phi^{\dagger}$ depends on $\Phi$ anti-linearly.

Indeed, $\Phi$ written in the Dirac notation as $\Phi=\lambda_{i j k l}\left|e_{i}\right\rangle \otimes\left\langle e_{j}|\otimes| e_{k}\right\rangle \otimes\left\langle e_{l}\right|$ is self-adjoint if and only if $\operatorname{Tr}(A \circ \Phi(B))=\operatorname{Tr}(\Phi(A) \circ B)$ for all $A, B \in \mathcal{L}_{2}(\mathcal{H})$, that applied to $A=\left|e_{j}\right\rangle \otimes\left\langle e_{i}\right|$ and $B=\left|e_{l}\right\rangle \otimes\left\langle e_{k}\right|$ yields $\lambda_{i j k l}=\lambda_{k l i j}$, so that we deal with maps coming from symmetric or antisymmetric tensors. What is more, since

$$
\mathcal{J}\left(\left|e_{i}\right\rangle \otimes\left\langle e_{j}|\otimes| e_{k}\right\rangle \otimes\left\langle e_{l}\right|\right)=\left|e_{i}\right\rangle \otimes\left\langle e_{l}|\otimes| e_{k}\right\rangle \otimes\left\langle e_{j}\right|,
$$

we have a further splitting

$$
\mathcal{L}_{2}^{s a}\left(\mathcal{H} \otimes \mathcal{H}^{*}\right)=\mathcal{L}_{2}^{s a+}\left(\mathcal{H} \otimes \mathcal{H}^{*}\right) \oplus \mathcal{L}_{2}^{s a-}\left(\mathcal{H} \otimes \mathcal{H}^{*}\right)
$$

where $\mathcal{L}_{2}^{s a \pm}\left(\mathcal{H} \otimes \mathcal{H}^{*}\right)$ consists of these $\Phi$ for which $\mathcal{J}(\Phi)= \pm \Phi$. Finally, we end up with bosonic and fermionic Jamiotkowski maps:

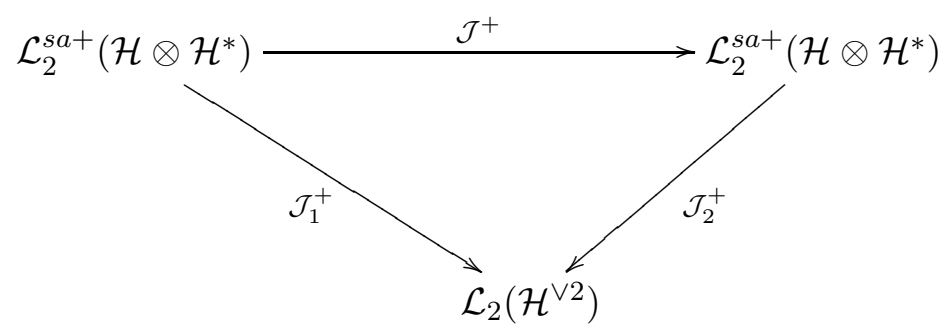

and

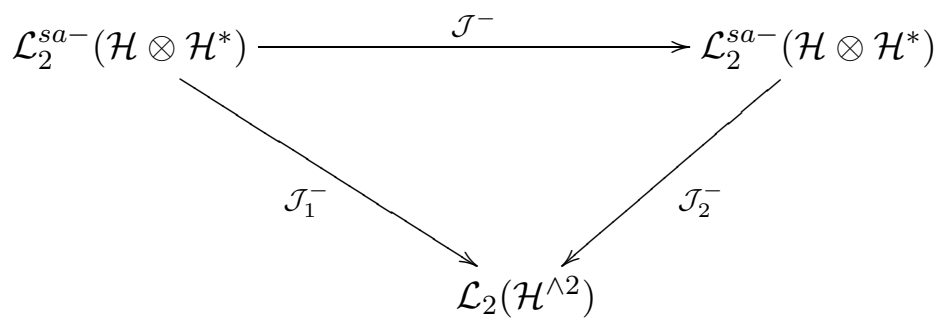

If now $\rho=|v\rangle\langle v|$ is a pure state in $\mathcal{H}^{\vee 2}$ corresponding to a vector $v \in \mathcal{H}^{\vee 2}$ with a Slater decomposition $v=\sum_{i=1}^{r} \lambda_{i} e_{i} \vee e_{i}, \lambda_{i}>0$, so that the Slater rank is $r$, then $\rho=\mathcal{J}_{1}^{+}(\Phi)$, with

$$
\Phi=\sum_{i, j} \lambda_{i} \lambda_{j}\left|e_{i}\right\rangle \otimes\left\langle e_{j}|\otimes| e_{i}\right\rangle \otimes\left\langle e_{j}\right|
$$

being a map from $\mathcal{L}_{2}^{s a+}\left(\mathcal{H} \otimes \mathcal{H}^{*}\right)$ of rank $r^{2}$.

Similarly, if $\rho=|w\rangle\langle w|$ is a pure state in $\mathcal{H}^{\wedge 2}$ corresponding to a vector $w \in \mathcal{H}^{\vee 2}$ with a Slater decomposition $w=\sum_{i=1}^{r} \mu_{i} f_{i} \wedge f_{n+i}, \mu_{i}>0$, so that the Slater rank is $r$, then $\rho=\mathcal{J}_{1}^{-}(\Phi)$, with

$$
\begin{aligned}
\Phi= & \sum_{i, j} \mu_{i} \mu_{j}\left(\left|f_{i}\right\rangle \otimes\left\langle f_{j}|\otimes| f_{n+i}\right\rangle \otimes\left\langle f_{n+j}|-| f_{n+i}\right\rangle \otimes\left\langle f_{j}|\otimes| f_{i}\right\rangle \otimes\left\langle f_{n+j}\right|\right. \\
& \left.-\left|f_{i}\right\rangle \otimes\left\langle f_{n+j}|\otimes| f_{n+i}\right\rangle \otimes\left\langle f_{j}|-| f_{n+i}\right\rangle \otimes\left\langle f_{n+j}|\otimes| f_{i}\right\rangle \otimes\left\langle f_{j}\right|\right)
\end{aligned}
$$

being a map from $\mathcal{L}_{2}^{s a-}\left(\mathcal{H} \otimes \mathcal{H}^{*}\right)$ of rank $4 r^{2}$. In this way we get a characterization of bosonic and fermionic simple pure states in terms of the corresponding Jamiołkowski isomorphisms. 
Theorem 7.1. A pure state $\rho$ in $\mathcal{H}^{\vee 2}$ (resp., $\mathcal{H}^{\wedge 2}$ ) is bosonic (resp., fermionic) simple if and only if $\rho=\mathcal{J}_{1}^{+}(\Phi)$ (resp., $\rho=\mathcal{J}_{1}^{-}(\Phi)$ ) for $\Phi \in \mathcal{L}_{2}^{s a+}\left(\mathcal{H} \otimes \mathcal{H}^{*}\right)$ (resp., $\Phi \in \mathcal{L}_{2}^{\text {sa- }}\left(\mathcal{H} \otimes \mathcal{H}^{*}\right)$ ) of rank 1 (resp., 4).

Remark 7.1. Of course, choosing a basis in $\mathcal{H}$ we can represent the above maps by matrices and gets the Jamiokkowski isomorphism in the form of permutation of indices, more familiar to physicists. The above form has the advantage that it does not depend on the basis, i.e. is canonical and covariant.

\section{Entangled states of composite systems with generalized paras- tatistics}

Our approach to the entanglement of composite systems for identical particles is so general and natural that it allows for an immediate implications also for generalized parastatistics.

Observe first that simple tensors of length 1 in $\tilde{\mathcal{H}}=\mathcal{H}_{1} \otimes \cdots \otimes \mathcal{H}_{k}$ form an orbit of the group $U\left(\mathcal{H}_{1}\right) \times \cdots \times U\left(\mathcal{H}_{k}\right)$ acting on $\tilde{\mathcal{H}}$ in the obvious way. In fact, each such tensor can be written as $e_{1}^{1} \otimes \cdots \otimes e_{1}^{k}$ for certain choice of orthonormal bases $e_{1}^{j}, \ldots, e_{n_{j}}^{j}$ in $\mathcal{H}_{j}, j=1, \ldots, k$. This means that simple tensors are just vectors of highest (or lowest - depending on the convention) weight of the compact Lie group $U\left(\mathcal{H}_{1}\right) \times \cdots \times U\left(\mathcal{H}_{k}\right)$ relative to some choice of a maximal torus and Borel subgroups. If indistinguishable particles are concerned, the symmetric and antisymmetric tensors in $\mathcal{H}^{k}$ form particular irreducible parts of the 'diagonal' representation of the compact group $U(\mathcal{H})$ in the Hilbert space $\mathcal{H}^{\otimes k}$, defined by

$$
U\left(x_{1} \otimes \cdots \otimes x_{k}\right)=U\left(x_{1}\right) \otimes \cdots \otimes U\left(x_{k}\right) .
$$

Recall that we identify the symmetry group $S_{k}$ with the group of certain unitary operators on the Hilbert space $\mathcal{H}^{k}$ in the obvious way,

$$
\sigma\left(x_{1} \otimes \cdots \otimes x_{k}\right)=x_{\sigma(1)} \otimes \cdots \otimes x_{\sigma(k)} .
$$

Note that the operators of $S_{k}$ intertwine the unitary action of $U(\mathcal{H})$. In the cases of the symmetric and antisymmetric tensors, we speak about Bose and Fermi statistics, respectively. But, for $k>2$, there are other irreducible parts of the representation (55), associated with invariant subspaces of the $S_{k}$-action, that we shall call (generalized) parastatistics. Any of these $k$-parastatistics (i.e. any irreducible subspace of the tensor product $\mathcal{H}^{\otimes k}$ ) is associated with a Young tableau $\alpha$ with $k$-boxes (chambers) as follows (see e.g. [22, 23]).

Consider partitions of $k: k=\lambda_{1}+\cdots+\lambda_{r}$, where $\lambda_{1} \geq \cdots \geq \lambda_{r} \geq 1$. To a partition $\lambda=\left(\lambda_{1}, \ldots, \lambda_{r}\right)$ is associated a Young diagram (sometimes called a Young frame or a Ferrers diagram) with $\lambda_{i}$ boxes in the $i$ th row, the rows of boxes lined up on the left. Define a tableau on a given Young diagram to be a numbering of the boxes by the integers $1, \ldots, k$, and denote with $Y_{\lambda}$ the set of all such Young tableaux. Finally, put $Y(k)$ to be the set of all Young tableaux with $k$ boxes. Given a tableau $\alpha \in Y(k)$ define two subgroups in the symmetry group $S_{k}$ :

$$
P=P_{\alpha}=\left\{\sigma \in S_{k}: \sigma \text { preserves each row of } \alpha\right\}
$$

and

$$
Q=Q_{\alpha}=\left\{\sigma \in S_{k}: \sigma \text { preserves each column of } \alpha\right\} .
$$

In the space of linear operators on $\mathcal{H}^{\otimes k}$ we introduce two operators associated with these subgroups:

$$
a_{\alpha}=\sum_{\sigma \in P} \sigma, \quad b_{\alpha}=\sum_{\sigma \in Q}(-1)^{\sigma} \sigma
$$


Finally, we define the Young symmetrizer

$$
c_{\alpha}=a_{\alpha} \circ b_{\alpha}=\sum_{\tau \in P, \sigma \in Q}(-1)^{\sigma} \tau \circ \sigma .
$$

It is well known that $\pi^{\alpha}=\frac{1}{\mu(\alpha)} c_{\alpha}$, for some non-zero rational number $\mu(\alpha)$, is an orthogonal projector and that the image $\mathcal{H}^{\alpha}$ of $c_{\alpha}$ is an irreducible subrepresentation of $U(\mathcal{H})$, i.e. the parastatistics associated with $\alpha$. As a matter of fact, these representations for Young tableaux on the same Young diagram are equivalent, so that the constant $\mu(\alpha)$ depends only on the Young diagram $\lambda$ of $\alpha$ (does not depend on the enumeration of boxes), $\mu(\alpha)=\mu(\lambda)$, and is related to the multiplicity $m(\lambda)$ of this irreducible representation in $\mathcal{H}^{\otimes k}$ by $\mu(\lambda) \cdot m(\lambda)=k$ ! For a given Young diagram (partition) $\lambda$, the map

$$
\epsilon_{\lambda}=\frac{1}{\mu(\lambda)^{2}} \sum_{\alpha \in Y_{\lambda}} c_{\alpha}
$$

is an orthogonal projection, called the central Young symmetrizer, onto the invariant subspace being the sum of all copies of the irreducible representations equivalent to that with a parastatistics from $Y_{\lambda}$.

The symmetrization $\pi^{\vee}$ (antisymmetrization $\pi^{\wedge}$ ) projection corresponds to a Young tableau with just one row (one column) and arbitrary enumeration. It is well known that any irreducible representation $\mathcal{H}^{\alpha}$ of $U(\mathcal{H})$ contains cyclic vectors which are of highest weight relative to some choice of a maximal torus and Borel subgroups in $U(\mathcal{H})$. We will call them $\alpha$-simple vectors or simple vectors in $\mathcal{H}^{\alpha}$. Note that such vectors can be viewed as generalized coherent states 24]. They were also regarded as the 'most classical' states by several authors [25]. These are exactly the tensors associated with simple (non-entangled) pure states for composite systems of particles with (generalized) parastatistics. This is because $\alpha$-simple tensors represent the minimal amount of quantum correlations for tensors in $\mathcal{H}^{\alpha}$ : the quantum correlations forced directly by the particular parastatistics.

Example 8.1. (a) For $k=2$ we have just the obvious splitting of $\mathcal{H}^{\otimes 2}$ into symmetric and antisymmetric tensors: $\mathcal{H}^{\wedge 2} \oplus \mathcal{H}^{\vee 2}$.

(b) For $k=3$, besides symmetric and antisymmetric tensors, we have two additional irreducible parts associated with the Young tableaux

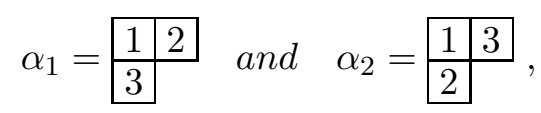

respectively. Hence,

$$
\mathcal{H}^{\otimes 3}=\mathcal{H}^{\wedge 3} \oplus \mathcal{H}^{\alpha_{1}} \oplus \mathcal{H}^{\alpha_{2}} \oplus \mathcal{H}^{\vee 3},
$$

with

$$
\begin{gathered}
\pi^{\alpha_{1}}: \mathcal{H}^{\otimes 3} \rightarrow \mathcal{H}^{\alpha_{1}}, \\
\pi^{\alpha_{1}}\left(x_{1} \otimes x_{2} \otimes x_{3}\right)=\frac{1}{3}\left(x_{1} \otimes x_{2} \otimes x_{3}+x_{2} \otimes x_{1} \otimes x_{3}-x_{3} \otimes x_{2} \otimes x_{1}-x_{3} \otimes x_{1} \otimes x_{2}\right),
\end{gathered}
$$

and

$$
\begin{gathered}
\pi^{\alpha_{2}}: \mathcal{H}^{\otimes 3} \rightarrow \mathcal{H}^{\alpha_{2}}, \\
\pi^{\alpha_{2}}\left(x_{1} \otimes x_{2} \otimes x_{3}\right)=\frac{1}{3}\left(x_{1} \otimes x_{2} \otimes x_{3}+x_{3} \otimes x_{2} \otimes x_{1}-x_{2} \otimes x_{1} \otimes x_{3}-x_{2} \otimes x_{3} \otimes x_{1}\right) .
\end{gathered}
$$


The simple tensors (the highest weight vectors) in $\mathcal{H}^{\alpha_{1}}$ can be written as

$$
v_{\lambda}^{\alpha_{1}}=\lambda\left(e_{1} \otimes e_{1} \otimes e_{2}-e_{2} \otimes e_{1} \otimes e_{1}\right),
$$

for certain choice of an orthonormal basis $e_{i}$ in $\mathcal{H}$ and $\lambda \neq 0$. Analogously, the simple tensors in $\mathcal{H}^{\alpha_{2}}$, in turn, take the form

$$
v_{\lambda}^{\alpha_{2}}=\lambda\left(e_{1} \otimes e_{2} \otimes e_{1}-e_{2} \otimes e_{1} \otimes e_{1}\right) .
$$

For $\operatorname{dim}(\mathcal{H})=3$, the simple tensors of length 1 form an orbit of the unitary group $U(\mathcal{H})$ of the (real) dimension 7 in $\mathcal{H}^{\alpha_{1}}$ and $\mathcal{H}^{\alpha_{2}}$. The simple symmetric tensors of length 1 form an orbit of the dimension 5, and the simple antisymmetric ones (of length 1) - an orbit of the dimension 1. The dimensions of the irreducible representations are: $\operatorname{dim}\left(\mathcal{H}^{\wedge 3}\right)=1, \operatorname{dim}\left(\mathcal{H}^{\vee 3}\right)=10$, $\operatorname{dim}\left(\mathcal{H}^{\alpha_{1}}\right)=\operatorname{dim}\left(\mathcal{H}^{\alpha_{2}}\right)=8$.

Let $\mathcal{H}^{\alpha} \subset \mathcal{H}^{\otimes k}$ denotes the irreducible component of the tensor representation of the unitary group $U(\mathcal{H})$ in $\mathcal{H}^{\otimes k}$ associated with a Young diagram $\alpha \in Y(k)$.

\section{Definition 8.1.}

(a) We say that a pure state $\rho \in \mathcal{H}^{\otimes k}$ obeys a parastatistics $\alpha \in Y(k)$ (is a pure $\alpha$-state in short) if $\rho$ is represented by a nonzero tensor $v \in \mathcal{H}^{\alpha}$, i.e.

$$
\rho=\rho_{v}=\frac{|v\rangle\langle v|}{\|v\|^{2}} .
$$

In other words, $\rho$ is a pure state on the Hilbert space $\mathcal{H}^{\alpha}$.

(b) A pure state $\rho \in \mathcal{H}^{\otimes k}$, obeying a parastatistics $\alpha$ is called a simple pure state for the parastatistics $\alpha$ (simple pure $\alpha$-state, in short), if $\rho$ is represented by an $\alpha$-simple tensor in $\mathcal{H}^{\alpha}$. If $\rho$ is not simple $\alpha$-state, we call it entangled pure $\alpha$-state.

(c) A mixed state $\rho$ on $\mathcal{H}^{\alpha}$ we call a simple (mixed) state for the parastatistics a (simple $\alpha$-state in short), if it can be written as a convex combination of simple pure $\alpha$-states. In the other case, $\rho$ is called entangled mixed $\alpha$-state.

For an arbitrary parastatistics (Young tableau) $\alpha \in Y(k)$ with the partition (Young diagram) $\lambda=\left(\lambda_{1}, \ldots, \lambda_{r}\right)$, consider the map

$$
i_{\alpha}: \mathcal{H}^{\times r} \rightarrow \mathcal{H}^{\otimes k}, \quad\left(x_{1}, \ldots, x_{r}\right) \mapsto x_{\alpha(1)} \otimes \cdots \otimes x_{\alpha(k)},
$$

where $\alpha(i)$ is the number of the raw in which the box with the number $i$ appears in the tableaux $\alpha$. In other words, we make a tensor product of $k$ vectors from $\left\{x_{1}, \ldots, x_{r}\right\}$ by putting $x_{j}$ in the places indicated by the number of the boxes in the $j$ th row. For instance, the Young tableaux from Example 8.1 give $i_{\alpha_{1}}\left(x_{1}, x_{2}\right)=x_{1} \otimes x_{1} \otimes x_{2}$ and $i_{\alpha_{2}}\left(x_{1}, x_{2}\right)=x_{1} \otimes x_{2} \otimes x_{1}$. It is clear that $i_{\alpha}\left(x_{1}, \ldots, x_{r}\right)$ is an eigenvector of $a_{\alpha}$.

It is easy to see now that the S-rank of the tensor $\pi^{\alpha}\left(x_{\alpha(1)} \otimes \cdots \otimes x_{\alpha(k)}\right)$, for $x_{1} \wedge \cdots \wedge x_{r} \neq 0$, is $r$ and that this is the minimal S-rank for tensors from $\mathcal{H}^{\alpha}$. Hence, the minimality of the S-rank is a good characteristic also for simple $\alpha$-tensors.

Theorem 8.1. A tensor $u \in \mathcal{H}^{\alpha}$ is $\alpha$-simple if and only if $u$ has the minimal S-rank among all non-zero tensors from $\mathcal{H}^{\alpha}$. This minimal $S$-rank equals $r$-the number of rows in the corresponding Young diagram. 


\section{Conclusions}

States of identical particles exhibit a priori correlations caused merely by (anti)symmetry of the wave function in the case of fermions or bosons. It is thus reasonable to treat as an analogue of the entanglement encountered in systems of distinguishable particles only an additional amount of correlation going beyond that stemming from symmetry requirements.

We proposed a way of treating all non-classical correlation, i.e., those which can be identified with the 'genuine entanglement' and not caused merely by symmetries. This unifies all cases: of distinguishable particles, fermions, and bosons, and can be easily extended to hypothetical multipartite systems consisting of particles subjected to arbitrary parastatistics.

We defined simple (non-entangled) pure states as one-dimensional selfadjoint projectors associated with simple tensors obeying appropriate symmetries identifying particles as bosons, fermions, etc. Consequently, simple (non-entangled) mixed states were defined as convex combinations of simple (non-entangled) pure ones. Such an unifying approach allowed also for description of such tools, known from the entanglement theory, as the Jamiołkowski isomorphism and Schmidt rank, to systems with other symmetries. The introduced concept of $S$-rank not only provides us with a tool for distinguishing entanglement of pure states with a given parastatistics, but is interesting also per se, as it offers the simplest characterization of highest weight vectors we know.

In the case of two fermionic subsystems our approach identifies non-entangled pure states to be the same as in all other approaches mentioned in Introduction, i.e., we identify them with simple antisymmetric tensors in the meaning explained in Section 4 above. In the bosonic case, from the geometric point of view, we clearly have a priori two inequivalent types of non-entanglement: tensor products of identical states and states associated with symmetrizations of products of orthogonal vectors. Non-entangled states of two different types are not connected by local unitary transformations which is in contrast to the familiar situation of distinguishable particles and intuitions build upon the fact that all separable states of distinguishable particles can be obtained from a single one by local transformations. Although this is obviously acceptable, it poses an open fundamental problem what is a physical meaning of two geometrically inequivalent types of non-entanglement.

In our approach we adopted the view that non-entangled pure bosonic states are simple symmetric tensors - tensor products of identical vectors. We find at least two arguments justifying this choice. In [7] it was pointed that all states which are symmetrizations of products of distinct vectors can be used to perform such clearly 'non-classical' tasks like teleportation. This definitely remains in conflict with the basic intuition connecting non-entanglement with the purely classical world. Second, from purely mathematical point of view, only tensor product of identical vectors provide highest weight vectors of the corresponding representation of the unitary group, like in the other cases.

Another achievements of our paper are explicit characterizations of simplicity (non-entanglement) for pure states in terms of directly verifiable, quadratic in coefficients, conditions which are computationally much easier than those proposed in the literature.

\section{References}

[1] E. Schrödinger. Discussion of probability relations between separated systems. Math. Proc. Cambridge Phil. Soc., 31:555-563, 1935. 
[2] F Herbut and M Vujičić. Irrelevance of the Pauli principle in distant correlations between identical fermions. J. Phys. A, 20(16):5555-5563, 1987.

[3] R Grobe, K Rzążewski, and J H Eberly. Measure of electron-electron correlation in atomic physics. J. Phys. B, 27(16):L503-L508, 1994.

[4] J. Schliemann, D. Loss, and A. H. MacDonald. Double-occupancy errors, adiabaticity, and entanglement of spin qubits in quantum dots. Phys. Rev. B, 63(8):085311, 2001.

[5] J. Schliemann, J. I. Cirac, M. Kuś, M. Lewenstein, and D. Loss. Quantum correlations in two-fermion systems. Phys. Rev. A, 64:022303, 2001.

[6] K. Eckert, J. Schliemann, D. Bruß, and M Lewenstein. Quantum correlations in systems of identical particles. Ann. Phys., 299:88-127, 2002.

[7] R. Paškauskas and L. You. Quantum correlations in two-boson wave functions. Phys. Rev. A, 64(4):042310, 2001.

[8] F. Herbut. How to distinguish identical particles. Am. J. Phys., 69(2):207-217, 2001.

[9] Y. S. Li, B. Zeng, X. S. Liu, and G. L. Long. Entanglement in a two-identical-particle system. Phys. Rev. A, 64(5):054302, 2001.

[10] G. Ghirardi, L. Marinatto, and T. Weber. Entanglement and Properties of Composite Quantum Systems: A Conceptual and Mathematical Analysis. Journal of Statistical Physics, 108(1):49-122, 2002.

[11] G. Ghirardi and L. Marinatto. General criterion for the entanglement of two indistinguishable particles. Phys. Rev. A, 70(1):012109, 2004.

[12] G. Ghirardi and L. Marinatto. Identical particles and entanglement. Optics and Spectroscopy, 99(3):386-390, 2005.

[13] T. Sasaki, T Ichikawa and I. Tsutsui. Entanglement of indistinguishable particles. Phys. Rev. A 83:012113, 2011.

[14] J. Grabowski, M. Kuś, and G. Marmo. Geometry of quantum systems: density states and entanglement. J. Phys. A: Math. Gen. 38:10217-10244, 2005.

[15] J. Grabowski, M. Kuś, and G. Marmo. Symmetries, group actions, and entanglement. Open Sys. Information Dyn., 13:343-362, 2006.

[16] R.A. Horn and C.R. Johnson. Matrix analysis. Cambridge University Press, 1985.

[17] I. Bengtsson and K. Życzkowski. Geometry of Quantum States. Cambridge University Press, 2006.

[18] A. Jamiołkowski. Linear transformations which preserve trace and positive semidefinite operators. Rep. Math. Phys. 3:275-278, 1972.

[19] M. D. Choi, Completely positive linear maps on complex matrices. Linear Alg. Appl. 10:285-278, 1975.

[20] J. Grabowski, M. Kuś, and G. Marmo. On the relation between states and maps in infinite dimensions. Open Sys. Information Dyn., 14:355-370, 2006. 
[21] P. Arrighi and C. Patricot. On quantum operations as quantum states. Ann. Phys. 311:26$52,2004$.

[22] W. Fulton and J. Harris. Representation Theory. A First Course. Springer Verlag, 1991.

[23] W. Fulton Young Tableaux. Cambridge University Press, 1997.

[24] A. Perelomov. Generalized coherent states and their applications. Springer, Heidelberg, 1986.

[25] M. Kuś and I. Bengtsson. Classical quantum states. Phys. Rev. A,80:022319, 2009. 